\title{
DOS NUEVAS ESPECIES DE PSEUDOSINELLA SCHÄFFER, 1897 (COLLEMBOLA, ENTOMOBRYIDAE) DEL GRUPO PETTERSENI PERTENECIENTES A LA COLECCIÓN BONET, CON CLAVES PARA SU DETERMINACIÓN
}

\author{
J. C. Simón Benito* \& J. G. Palacios Vargas**
}

\begin{abstract}
RESUMEN
Se describen dos nuevas especies Pseudosinella Schäffer, 1934 del grupo petterseni, con ejemplares de la colección de Federico Bonet: P. parattenuata sp. n. de los Estados Unidos y P. federicoi sp. n. de Argentina. Se proporciona una clave mundial para todas las especies que presentan un grueso diente en el empodio.

Palabras clave: Collembola, Pseudosinella, Argentina, especies nuevas.
\end{abstract}

\begin{abstract}
Two new species of Pseudosinella Schäffer, 1897 (Collembola, Entomobryidae) of the petterseni group from the Bonet collection, with keys for its identification

Two new species of Pseudosinella Schäffer, 1897 from the Bonet collection are described: Pseudosinella parattenuata $\mathbf{s p}$. n. from USA and P. federicoi $\mathbf{s p .}$. n. from Argentina, both are established in the petterseni group, with specimens of Federico Bonet's collection. A key for the world species of the genus with a thick tooth in the empodium is given.

Keywords: Collembola, Pseudosinella, Argentina, new species.
\end{abstract}

\section{Introducción}

Bonet en 1934 publica una serie de especies de colémbolos cavernícolas, recogidos por C. Bolívar y R. Jeannel, de cuevas de América del Norte. Entre ellas establece un nuevo taxón, Pseudosinella pet- terseni var. attenuata, caracterizado por su espolón puntiagudo y de menor longitud que la uña. Este taxon se describe aquí como una especie nueva: $P$. parattenuata $\mathbf{s p .} \mathbf{n}$.

Además, los 3 ejemplares de Argentina nominados como $P$. petterseni por Bonnet en 1933, no

* Universidad Autónoma de Madrid, Facultad de Ciencias, Departamento de Biología, Unidad Docente de Zoología, C/ Darwin, n. 2, Cantoblanco, 28049 Madrid. e-mail: carlos.simon@uam.es.

** Laboratorio de Ecología y Sistemática de Microartrópodos, Depto. Ecología y Recursos Naturales, Fac.Ciencias, UNAM, 04510, México D. F., México. e-mail: troglolaphysa@hotmail.com. 
corresponden a la redescripción de esta especie efectuada por Gisin en 1967, por lo describen como P. federicoi sp. n.

Se ha seguido la nomenclatura de sedas establecida por Gisin (1967) y modificada por Christiansen et al. (1990) y para la separación de especies nos hemos guiado por las claves que da dicho autor en 2007 en internet.

\section{Pseudosinella federicoi sp. n.}

Pseudosinella petterseni, Bonet, 1933 nec Börner, 1901

Material tiPo: Argentina, Buenos Aires, La Plata, 1932, J.A. Rosas leg, 1 ejemplar en la preparación nominada como: $927 \mathrm{~N}$ (Holotipo); 1 ejemplar en la preparación nominada: $927 \mathrm{aN} ; 1$ ejemplar en la preparación nominada: $927 \mathrm{bN}$. El material está depositado en el Museo Nacional de Ciencias Naturales, Madrid, CSIC (Cat. Tipos MNCN No 9986).

DESCRIPCIÓN. Longitud máxima $1,4 \mathrm{~mm}$. Sin pigmento en el cuerpo, sin ojos. Antenas sin pigmento, su relación con la diagonal cefálica varía de 1,60 a 1,75. La relación de los artejos antenales I:II:III:IV es 13:45:23:55, tercer artejo antenal con la mitad de la longitud que el segundo. No se ha observado ninguna vesícula en el IV artejo antenal. El órgano sensorial del III artejo antenal está formado por dos bastoncitos ligeramente inclinados.

Cabeza sin R0, sin R1, R2, ni R3, con un botriotrico en la región postero-lateral de la cabeza junto a una macroseda, la longitud de esta última, con respecto al botriotrico, es de un 33\%, existen dos inserciones más, pero se corresponden con bases de inserción de escamas (Fig. 1). Fórmula labial: $m_{1}$ $\mathrm{m}_{2} \mathrm{r}$ e $1_{1} 1_{2}$ todas lisas, $\mathrm{r}$ es una microseda (Fig. 4), con 4 sedas lisas a lo largo del surco cefálico ventral, seda de la papila labial externa con una seda curva que alcanza el ápice de la papila, con 3 sedas sublobulares en el lóbulo maxilar y una seda recta que alcanza el ápice de la papila, con 4 papilas labrales, las dos internas reducidas y setáceas.

Tibiotarso con un espolón puntiagudo, su relación con la longitud de la uña es de 0,73 . Uña con un diente mediano interno situado en el $45 \%$ de su longitud interna, existe un par basal situado proximalmente, uno de ellos dos veces más desarrollado que el otro y su ápice alcanza el $31 \%$ de la longitud de la uña, el más basal de menor tamaño se localiza en el $26 \%$ de la misma. Apéndice empodial con un gran diente interno situado aproximadamente en la mitad que la longitud del mismo (Fig. 5). Patas sin escamas, las sedas internas del tibiotarso son ciliadas y acabadas en punta.

La fórmula de las macrosedas dorsales es: 0001/00/0301(0)+2. Quetotaxia del II segmento abdominal: $\mathrm{pABQ}_{1} \mathrm{q}_{2}$ la relación de las macrosedas A:B:Q es 30:90:110 (Fig. 2). El segmento abdominal IV presenta una mesoseda anterior $\left(\mathrm{P}_{1}\right)$, su relación con la medial es 100:25 (algunos autores toman a esta seda como macroseda, otros no, por lo que hay una gran confusión en su definición) (Fig. 3). La macroseda $M_{1}$ sale algo por debajo del botriotrico, sin seda accesoria "s" y con seda e, ésta se inserta un poco por encima del botriotrico.

Tubo ventral con $5+5$ sedas distales lisas y $2+2$ en la región medial posterior. Retináculo con $4+4$ dientes, se ha visto una seda, pero puede que existan más. Manubrio distalmente con una seda interna y 2 externas junto a los dos pseudoporos. Mucrón bidentado y con una seda en su base.

Etimología. Dedicamos esta especie en homenaje al Dr. Federico Bonet Marco, colembólogo español, indiscutible referencia para los que se han

Tabla 1.- Caracteres diferenciadores entre las especies que presentan igual macroquetotaxia dorsal, ausencia de ojos, presencia de un gran diente en el empodio y sedas del labio lisas.

Table 1.- Differential characters between species with the same dorsal macrochaetotaxy, no eyes, presence of a large tooth in the empodium appendage, and smooth setae in the labium.

\begin{tabular}{|c|c|c|c|c|c|c|c|}
\hline Especies Autor y año & $\begin{array}{c}\text { Seda } r \\
\text { del labio }\end{array}$ & s IV & $\begin{array}{l}\text { Diente par } \\
\text { basal uña }\end{array}$ & $\begin{array}{l}\text { Diente par } \\
\text { distal uña }\end{array}$ & $\begin{array}{c}\text { Diente impar } \\
\text { uña }\end{array}$ & $\begin{array}{c}\text { Espolón } \\
\text { puntiagudo }\end{array}$ & $\begin{array}{c}\text { Longitud } \\
\text { uña/espolón }\end{array}$ \\
\hline ashmoleorum Gama, 1988 & + & - & $31 \%$ & $45 \%$ & $-+20 \%$ ?* & + & 0,55 \\
\hline federicoi $\mathbf{s p .} \mathbf{n}$. & + & - & $26 \%$ & $31 \%$ & $45 \%$ & + & 0,73 \\
\hline gamae Gisin, 1967 & + & + & $42 \%$ & $51 \%$ & $60-62 \%$ & - & 0,57 \\
\hline \multicolumn{8}{|l|}{ violenta sensu Christiansen } \\
\hline \& Bellinger, 1998 & - & + & $45 \%$ & $55 \%$ & $\begin{array}{c}68-78 \% \\
\text { o sin diente interno }\end{array}$ & +- & 1 \\
\hline
\end{tabular}

*en la uña Gama 1988 no dibuja el diente interno, a lo más existe un abultamiento que podría ser considerado como tal, situado en el $20 \%$ de la longitud de su cresta interna (Fig. 6). 


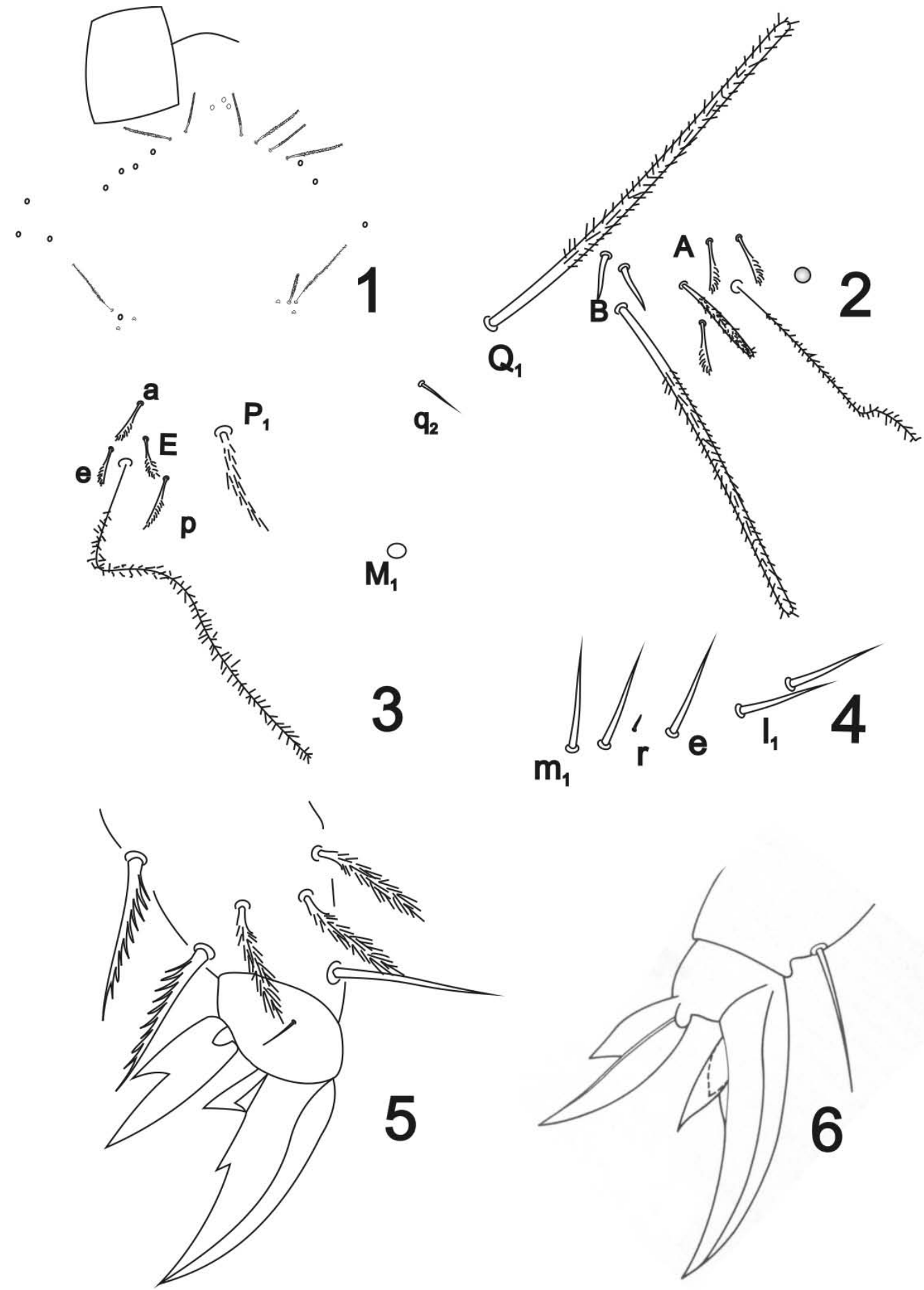

Figs. 1-6.- 1-5) Pseudosinella federicoi sp. n. 1) Quetotaxia cefálica, 2) Quetotaxia del segmento abdominal II, 3) Quetotaxia del segmento abdominal IV, 4) Quetotaxia del labio, 5) Uña del par de patas III. 6) Pseudosinella ashmoleorum Gama, 1988. Uña del III par de patas.

Figs. 1-6.- 1-5) Pseudosinella federicoi sp. n. 1) Chaetotaxy of the head, 2) Chaetotaxy of abdominal segment II, 3) Chaetotaxy of abdominal segment IV, 4) Labial Chaetotaxy, 5) Unguis III. 6) Pseudosinella ashmoleorum Gama, 1988. Unguis III. 
iniciado en el estudio de este grupo de hexápodos en España. Revisó con minuciosidad gran cantidad de especies, en particular del género Pseudosinella, y describió varias para la ciencia. Continuó su trabajo en México a partir de los años 40 del pasado siglo, sentando las bases para su estudio, y es el padre de la Bioespeleología en dicho país.

Discusión. Pseudosinella federicoi $\mathbf{s p .} \mathbf{n}$. es una especie muy próxima a $P$. ashmoleorum Gama 1988, P. gamae Gisin, 1967 y P. violenta (Folsom, 1924) sensu Christiansen \& Bellinger (1998), por tener una macroquetotaxia igual, ausencia de ojos, sedas del labio MMELL lisas y un gran diente en el apéndice empodial. Las diferencias se dan en la Tabla 1.

\section{Pseudosinella parattenuata sp. $\mathbf{n}$.}

Pseudosinella petterseni var. attenuata Bonet, 1934 (no disponible)

MATERIAL. USA. Virginia, Luray Cavern, 1928, 1 ejemplar en la preparación nominada como $590 \mathrm{~N}$, leg. C. Bolívar y R. Jeannel; 1 ejemplar en la preparación nominada como $590 \mathrm{aN}$ (Holotipo), leg C. Bolívar y R. Jeannel. El material está depositado en el Museo Nacional de Ciencias Naturales, Madrid, CSIC, y determinado por F. Bonet como Pseudosinella petterseni var. attenuata nov.

DESCRIPCIÓN. Longitud de 1,1 a 1,4 mm. Sin pigmento en el cuerpo, sin ojos, no se ha podido determinar la longitud de las antenas debido a que éstas se encuentran incompletas, por esta razón no se puede afirmar la presencia de vesícula apical en el segmento antenal IV, ni la morfología (o la estructura) del órgano sensorial del artejo antenal III.

Cabeza sin ojos. Cabeza con R0, R1 y R3, carece de seda R2, sin seda S, con sedas T, Po. Labio con sedas M, E y L ciliadas, no se ha podido observar con claridad la presencia de seda $r$, en su lugar existe un artefacto que no deja ver con nitidez su existencia, la fórmula por lo tanto seria $\mathrm{M}_{1} \mathrm{M}_{2} \mathrm{r}$ ? E $\mathrm{L}_{1} \mathrm{~L}_{2}$. (Fig. 7). Con 4 sedas a lo largo del surco cefálico ventral, seda de la papila labial externa curva, alcanza el ápice de la misma, lóbulo maxilar externo con 3 sedas sublobulares, con 4 papilas en el labro, grandes y claras.

Tibiotarso con un espolón puntiagudo de menor tamaño que la longitud de la uña, su relación es: 0,68 . La uña con un diente mediano interno situado en el $17,7 \%$ de la longitud de su cresta interna, existe un par basal situado proximalmente, con un diente más del doble que el otro y su ápice alcanza el $24,1 \%$ de dicha cresta interna, el más basal de menor tamaño y alcanza en el $24,8 \%$ de la misma (Fig. 10). Apéndice empodial con un gran diente interno situado aproximadamente de la mitad de la longitud del margen interno del empodio. Patas sin escamas, el tibiotarso presenta macrosedas ciliadas y puntiagudas.

La fórmula de las macrosedas dorsales es: R011/40/0101(0)+2?. Quetotaxia del segmento abdominal II: $\mathrm{paBq}_{1} \mathrm{q}_{2}$ (Fig. 9).

En el segmento abdominal IV no se observa ninguna macroseda anterior, en su lugar existe una mesoseda $\left(\mathrm{P}_{1}\right)$, el doble de la longitud de la seda más cercana (Fig. 8), algunos autores han optado por considerar que tal seda, aunque de menor tamaño, es una macroseda, así lo hace Gisin (1967) para P. gamae, mientras que Gama (1988) mantiene el criterio contrario para esta misma seda, cuando describe $P$. ashmoleorum. Aquí se ha optado por mantener los dos criterios a efectos prácticos y tratar de uniformizar todas las especies que presentan esta característica.

Tabla 2.- Caracteres diferenciadores entre las especies que presentan un gran diente en el empodio, espolones puntiagudos, con macrosedas en el segmento torácico II y ausencia de ojos.

Table 2.- Differential characters between species that have a large tooth in empodium appendage, sharp tennent hair, with macrosetae in thorax segment II and the absence of eyes.

\begin{tabular}{|c|c|c|c|c|c|c|c|}
\hline Especies Autor y año & $\begin{array}{l}\text { Macrosedas } \\
\text { dorsales }\end{array}$ & $\mathbf{T}$ & $\begin{array}{c}\text { Labio } \\
\text { fórmula }\end{array}$ & $\begin{array}{l}\text { Sedas MMELL } \\
\text { ciliadas }\end{array}$ & II Ab & Seda $Q$ & s IV \\
\hline parattenuata $\mathbf{s p .} \mathbf{n}$. & $\mathrm{R} 011 / 40 / 0101(0)+2 ?$ & + & M1M2r?EL1L2 & + & $\mathrm{paBq} 1 \mathrm{q} 2$ & - & + \\
\hline anderseni Gisin, 1967 & $\mathrm{R} 00 / 10 / 0201+2$ & - & M1M2rEL1L2 & + & $\mathrm{pABq} 1 \mathrm{q} 2$ & - & + \\
\hline certa Christiansen \& Bellinger, 1980 & $\mathrm{R} 00 / 10 / 0100+2$ & - & M1M2-EL1L2 & + & $\mathrm{paBq} 1 \mathrm{q} 2$ & - & + \\
\hline gajui Luciáñez \& Simón, 1994 & $\mathrm{R} 011 / 32 / 0101+2$ & + & M1M2rEL1L2 & + & $-a B q 1 q 2$ & - & + \\
\hline
\end{tabular}




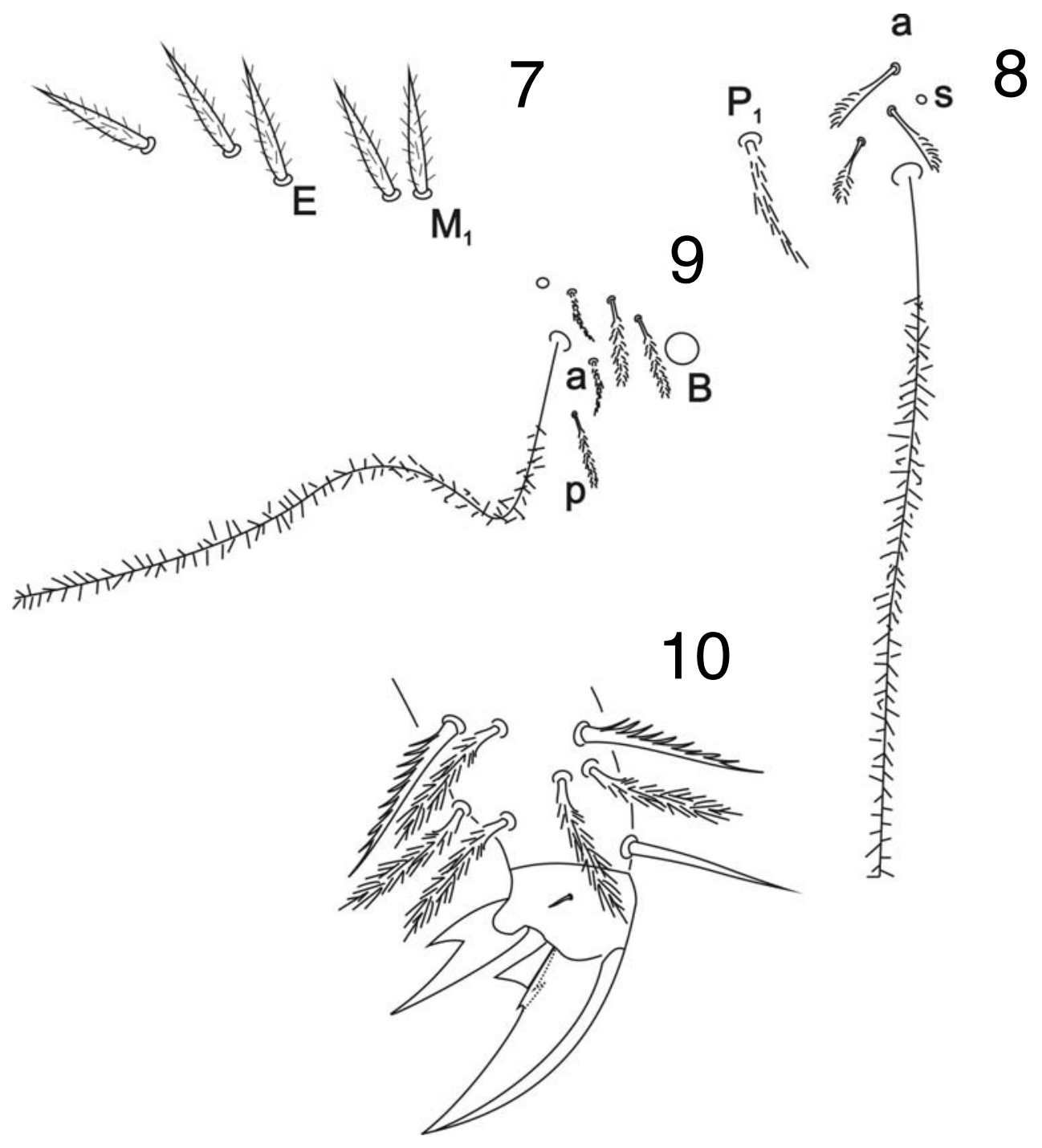

Figs. 7-10.- Pseudosinella parattenuata sp. n. 7) Quetotaxia del labio, 8) Quetotaxia del segmento abdominal IV, 9) Quetotaxia del segmento abdominal II, 10) Uña del III par de patas.

Figs. 7-10.- Pseudosinella parattenuata sp. n. 7) Labial Chaetotaxy, 8) Chaetotaxy of abdominal segment IV, 9) Chaetotaxy of abdominal segment II, 10) Unguis III.

Con seda accesoria "s" junto al botriotrico anterior. En los ejemplares estudiados existe una gran cantidad de productos de la digestión que impiden observar con claridad los detalles quetotáxicos, se ve la base de inserción de la macroseda mediana posterior, mientras que la de la anterior no se aprecia, de ahí el signo de interrogación en la fórmula quetotáxica para este segmento, 1(0)+2? (Fig. 8).
Tubo ventral con $5+5$ sedas distales lisas y $2+2$ en la región medial posterior. Retináculo con $4+4$ dientes, al menos con una seda. Las sedas del manubrio no se aprecian con claridad, debido al estado de la preparación. Mucrón bidentado y con una seda en su base.

ETIMología. El nombre de parattenuata se elige en honor del Dr. Federico Bonet Marco, ya que él nominó a este taxon attenuata. 
Discusión. Este taxón es único por su macroquetotaxia dorsal y no se parece a ningún otro, por lo que se eleva a categoría de especie, a pesar de haber sido descrita como variedad de petterseni, y se aleja claramente de ella en el número y disposición de las macrosedas dorsales. Por la ausencia de ojos en la cabeza, presencia de un gran diente interno en el apéndice empodial, tibiotarsos con espolones puntiagudos y con macrosedas torácicas se aproxima a las especies incluidas en la tabla 2.

El nombre de variedad dado por Bonet en 1934 no está disponible (ICZN, Arts. 45.5 y 45.6) al tratarse de un término infrasubespecífico, puesto que en el mismo trabajo se describen subespecies.

Clave mundial de las especies del género Pseudosinella con un grueso diente en el apéndice empodial (grupo petterseni)

1. Segmento abdominal IV con una macroseda P muy clara ..... .14

- Segmento abdominal IV sin una macroseda P clara, o microseda

2. Cabeza con $6+6$ ojos

- Cabeza con menos ojos

kenchristianseni Gruia, 1984

3. Con $4-5$ ojos lahainaensis Christiansen \& Luther, 1986

- Sin ojos ... 4

4. Tórax II con macrosedas ……………………...... 5

- Tórax II sin macrosedas ........................................... 6

5. Cabeza con seda $T$

- Cabeza sin seda T

parattenuata sp. $\mathbf{n}$. certa Christiansen \& Bellinger, 1980

6. Abdomen II con 3 macrosedas 11

- Abdomen II con menos de 3 macrosedas 7

7. Abdomen II con 2 macrosedas

- Abdomen con 1 macroseda sera Christiansen \& Bellinger, 1980

8. La seda $\mathrm{q}_{1}$ del II segmento abdominal es una microseda

- La seda $\mathrm{Q}_{1}$ del II segmento abdominal es una macroseda

9. Sedas MMELL del labio ciliadas pseudopetterseni Jordana \& Baquero, 2007

- Sedas mmell del labio lisas rolfsi sensu Christiansen \& Bellinger, 1998

10. Cabeza con seda $T$ biungiculata sensu Mari Mutt, 1986

- Cabeza sin seda T fujiokai Yosii, 1964
11. Tibiotarsos con espolones mazudos 13

- Tibiotarsos con espolones puntiagudos 12

12. Diente par basal de la uña situado en el $26 \%$ de su longitud, el distal en el $31 \%$ de la misma, con un diente impar situado en el 45\%. Espolón de 3/4 la longitud de la uña federicoi sp. $\mathbf{n}$.

- Diente par basal de la uña situado en el $31 \%$ de su longitud, el distal en el $45 \%$ de la misma, a veces presenta diente impar. Espolón de 1/2 la longitud de la uña ashmoleorum Gama, 1988

13. Labio con seda $r$ gamae Gisin, 1967

- Labio sin seda $r$ violenta (Folsom, 1924)

14. Tórax con macrosedas ........................................... 19

- Tórax sin macrosedas .......................................... 15

15. Abdomen II con seda $\mathrm{p}$...................................... 16

- Abdomen II sin seda $\mathrm{p}$.......................................... 17

16. Sin ojos hrabrei Rusek, 1979

- Cabeza con 4+4 ojos ....... azerbaidzhani Tshelnokov \& Rasulova, 1982

17. Tibiotarsos con espolones mazudos bellingeri Wang, Christiansen \& Chen, 2002

- Tibiotarsos con espolones puntiagudos 18

18. Con seda $r$ en el labio ..maros Deharveng \& Suhardjono, 2004

- Sin seda $\mathrm{r}$ en el labio caoi Chen, Wang \& Christiansen, 2002

19. Tórax II con una macroseda ................................ 20

- Tórax II con más macrosedas .............................. 26

20. Cabeza con $6+6$ ojos charoae Villanueva \& Jordana, 1986

- Con menos ojos en la cabeza 21

21. Abdomen II con 3 macrosedas baghdadica Rusek, 1981

- Abdomen II con menos macrosedas ................... 22

22. Abdomen II con 2 macrosedas .............................. 23

- Abdomen II con una macroseda ……………...... 24

23. Algunas de las sedas del labio $\mathrm{m} / \mathrm{e} / \mathrm{l}$ lisas seyleri Stomp \& Tommasi-Ursone, 1989

- Todas las sedas del labio MMELL ciliadas anderseni Gisin, 1967

24. Con $2+2$ ojos .... cobosae Luciáñez \& Simón, 1994

- Con $3+3$ ojos 25

25. Abdomen IV sin seda $\mathrm{s}$ espagnoli Simón \& Selga, 1977

- Abdomen IV con seda s arretzi Simón, 1979

26. Torax II-III con 2-2 macrosedas petterseni Börner, 1901

- Torax II-III con otra quetotaxia 27 
27. Torax III con una macroseda espana Christiansen, 1961

- Tórax III con otra quetotaxia 28

28. Tórax III con 3 macrosedas 29

- Tórax III sin macrosedas soriensis Simón \& Signoret, 2006

29. Sedas MMELL todas ciliadas

bachae Luciáñez \& Simón, 1994

- Con alguna seda mmell del labio lisa espanita Christiansen \& Bellinger, 1996

No se han incluido en la clave $P$. difficilis Denis, 1926, ni P. halophila Bagnall, 1939, por lo incompleto de sus descripciones.

\section{AGRADECIMIENTOS}

Al Dr. Kenneth A. Christiansen (Grinnell College, Iowa, EEUU) por enviarnos ejemplares determinados como $P$. violenta y P. rolfsi. A Isabel Izquierdo y Carolina Martín (Museo de Historia Natural, Madrid, España) excelentes amigas y colaboradoras en la gestión de las colecciones de este grupo de microartrópodos.

\section{Referencias}

Bonet, F., 1933. Colémbolos de la República Argentina. Eos, 9: 123-194.

Bonet, F., 1934. LX. Campagne spéleologique de C. Bolívar et R. Jeannel dans l'Amérique du Nord. Archives de Zoologie Expérimentale et Génerale, 76: 361-377.

Christiansen, K., 2007. NaviKey 2.3 for Collembola dataset.

http://www.math.grin.edu/ twitchew/coll/navikey.html
Christiansen, K. \& Bellinger, P., 1998. The Collembola of North America, North of Rio Grande, A taxonomic analysis, part 3, Family, Entomobryidae. Grinnell College. Grinnell. pp. 877-1174.

Christiansen, K., Bellinger, P. \& Gama, M. M., 1990. Computer assisted identification of specimens of Pseudosinella (Collembola Entomobryidae). Revue d'Ecologie et Biologie du Sol, 27: 231-246.

Gama, M. M., 1988. Systématique évolutive des Pseudosinella. XIV. Deux espéces nouvelles provenant des Açores (Insecta: Collembola). Revue Suisse de Zoologie, 95: 607-611.

GisIn, H., 1967. Espèces nouvelles et lignées évolutives de Pseudosinella endogés (Collembola). Memórias e Estudos do Museu Zoológico da Universidade de Coimbra, 301: 5-25. 